\title{
Gravity-driven slug motion in capillary tubes
}

\author{
Ivan Lunati ${ }^{1, a)}$ and Dani Or $^{2}$ \\ ${ }^{1}$ School of Architecture, Civil and Environmental Engineering, EPF Lausanne, Switzerland \\ ${ }^{2}$ Department of Environmental Sciences, ETH Zurich, Switzerland
}

(Received 11 February 2008; accepted 1 April 2009; published online 14 May 2009)

\begin{abstract}
The velocity of a liquid slug falling in a capillary tube is lower than predicted for Poiseuille flow due to presence of menisci, whose shapes are determined by the complex interplay of capillary, viscous, and gravitational forces. Due to the presence of menisci, a capillary pressure proportional to surface curvature acts on the slug and streamlines are bent close to the interface, resulting in enhanced viscous dissipation at the wedges. To determine the origin of drag-force increase relative to Poiseuille flow, we compute the force resultant acting on the slug by integrating Navier-Stokes equations over the liquid volume. Invoking relationships from differential geometry we demonstrate that the additional drag is due to viscous forces only and that no capillary drag of hydrodynamic origin exists (i.e., due to hydrodynamic deformation of the interface). Requiring that the force resultant is zero, we derive scaling laws for the steady velocity in the limit of small capillary numbers by estimating the leading order viscous dissipation in the different regions of the slug (i.e., the unperturbed Poiseuille-like bulk, the static menisci close to the tube axis and the dynamic regions close to the contact lines). Considering both partial and complete wetting, we find that the relationship between dimensionless velocity and weight is, in general, nonlinear. Whereas the relationship obtained for complete-wetting conditions is found in agreement with the experimental data of Bico and Quéré [J. Bico and D. Quéré, J. Colloid Interface Sci. 243, 262 (2001)], the scaling law under partial-wetting conditions is validated by numerical simulations performed with the Volume of Fluid method. The simulated steady velocities agree with the behavior predicted by the theoretical scaling laws in presence and in absence of static contact angle hysteresis. The numerical simulations suggest that wedge-flow dissipation alone cannot account for the entire additional drag and that the non-Poiseuille dissipation in the static menisci (not considered in previous studies) has to be considered for large contact angles. () 2009 American Institute of Physics.
\end{abstract}

[DOI: $10.1063 / 1.3125262$ ]

\section{INTRODUCTION}

The motion of a finite liquid volume constrained between solid surfaces is governed by a complex interplay of capillary, viscous, and gravitational forces. For sufficiently long slugs, capillary forces are negligible and the flow is satisfactorily described by Poiseuille law with parabolic velocity profile across the tube, and the mean velocity is proportional to the cross-sectional area and to the applied pressure gradient (see, e.g., Ref. 1). In contrast, short slugs are slower than predicted for Poiseuille flow due to the role played by capillary forces. We address the problem of determining the steady velocity of a finite liquid slug falling in a capillary tube. Despite the simple geometry, this problem retains the complexity of three-force interactions: viscosity and gravity modify the meniscus shapes, which, in turn, changes the capillary pressure acting on the interface.

Several studies have addressed the dependence of the contact angle on the velocity. Usually it is assumed that, in a small wedge close to the moving contact line, friction at the solid surface and capillary forces at the liquid-gas interface

\footnotetext{
${ }^{\text {a) }}$ Author to whom correspondence should be addressed. Laboratory of Environmental Fluid Mechanics, EPFL-ENAC-EFLUM, Batiment GR (A0 455), Station 2, 1015 Lausanne, Switzerland. Telephone: +41-21-693 6369. Electronic mail: ivan.lunati@epfl.ch
}

are the dominant forces and must balance at steady state. This description has been applied to spontaneous wetting, ${ }^{2-4}$ and to the problem of a plate pulled from or pushed into a liquid bath. ${ }^{5-7}$ In these cases, the bulk fluid is considered at rest and gravity is negligible up to a distance from the contact line equal to capillary length. ${ }^{8}$ In the case of forced wetting ${ }^{9,10}$ the velocity is not solely determined by viscous and capillary forces: the forcing term (e.g., the force applied by variable speed motor in the classical experiment of Hoffman ${ }^{9}$ ) plays a primary role and must be included in the wedge force balance. This can be done by relating the curvature of the free surface to the viscous pressure at the liquid-vapor interface in a wedgelike geometry, which depends both on the wall friction and on the pressure forces exerted by the bulk liquid. ${ }^{11,12}$

In this paper, we consider gravity-driven slug flow in a capillary and derive scaling relationships for its steady velocity. Previous studies addressing similar problems tacitly assume that friction is essentially due to Poiseuille viscous dissipation. ${ }^{13-15}$ This implies that the additional drag force is only due to the altered meniscus shapes that modify the applied pressure. However, the presence of an interface also modifies the flow field (bending streamlines in the vicinity of the meniscus) and increases viscous dissipation. In Sec. II, through the use of a formula from the differential geometry, 


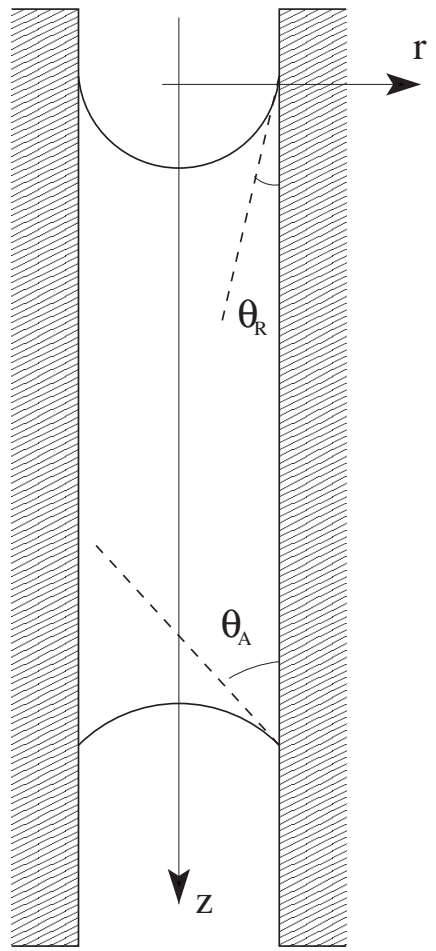

FIG. 1. A liquid slug of length $L$ is confined in a capillary tube of radius $b$. The advancing, respectively receding, meniscus has surface $S_{A}$, respectively, $S_{R}$, and meets the wall at an angle $\theta_{A}$, respectively, $\theta_{R}$.

we rigorously separate the effects of capillary pressure from those of friction and demonstrate that there is no capillary drag of hydrodynamic origin (i.e., due to hydrodynamic deformation of the menisci). In Sec. III, we recall some classical models that we use to estimate the shape of the menisci near contact lines and determine the associated increase in viscous friction. In Sec. IV, we combine the results of the previous sections and obtain scaling laws for the velocity of a steadily falling liquid slug. The theoretical scaling laws are validated against results from numerical simulations in Sec. $\mathrm{V}$; and the main results are discussed in Sec. VI.

\section{RESULTANT OF THE FORCES ACTING ON A SLUG}

Let us consider a liquid slug of volume $V$, which is confined in a capillary tube of radius $b$ (Fig. 1). The acceleration is determined by the force resultant, which is obtained by integration of the Navier-Stokes equations over the liquid volume $V$. At steady state, the resultant must be zero and the integral takes the form

$$
-\int_{S} p n_{j} d s+\mu \int_{S} \frac{\partial u_{j}}{\partial x_{k}} n_{k} d s+\rho V g_{j}=0,
$$

where $p$ is the liquid pressure; $\boldsymbol{u}$ is the velocity; $\boldsymbol{n}$ the normal to the surface of the liquid $S=\partial V ; \boldsymbol{g}$ the gravity acceleration; $\rho$ and $\mu$ the density and the viscosity of the liquid, respectively. The first term in Eq. (1) describes the effects of external pressure forces, the second term the contribution of viscous stresses, and the third term accounts for the body force.

If gravitational acceleration is aligned with the capillary axis $(z)$, only the resultant component in the $z$-direction is nonzero, hence, only the pressure forces acting on the two menisci and the viscous forces acting at the capillary walls contribute to the resultant. Neglecting viscous effects at the free surface, the liquid pressure is given by Laplace formula, $p=p_{0}+\kappa \gamma$, where $p_{0}$ is the uniform gas pressure (equal on both sides of the slug), $\gamma$ is the surface tension, and $\kappa$ is the curvature (positive if the center of curvature lies in the liquid). Through the use of Frenet's formulas (see, e.g., Ref. 16),

$$
\int_{S} \kappa n_{j} d s=\oint_{\partial S} t_{j} d l
$$

( $t$ is the conormal vector), we write the first integral in Eq. (1) as

$$
\begin{aligned}
\int_{S} p n_{j} d s & =\int_{S_{R}} \gamma \kappa n_{j} d s+\int_{S_{A}} \gamma \kappa n_{j} d s \\
& =\gamma\left(\cos \theta_{R}-\cos \theta_{A}\right) \oint_{\partial S} d l,
\end{aligned}
$$

where $S_{R}$ and $S_{A}$ indicates the surfaces of the receding and advancing meniscus, respectively; $\theta_{A}$, respectively, $\theta_{R}$, is the advancing, respectively, receding, contact angle; and $\oint_{\partial S} d l$ is the length of the contact line.

Equation (3) is valid as long as interface forces are proportional to the surface curvature, that is, down to a distance from the solid wall comparable with the molecular size: closer to the wall the hydrodynamic description breaks down and intermolecular forces dominate. Therefore, $\theta_{R}$ and $\theta_{A}$ in Eq. (3) are the microscopic contact angles whose determination falls outside the scope of hydrodynamics. In the following we will assume that these two angles are independent of the absolute velocity: $\theta_{R}$ and $\theta_{A}$ remain equal to the static receding and static advancing contact angles, respectively.

Introducing the equivalent slug length $L$ (slug volume, $V$, divided by cross-sectional area) and the velocity of the center of mass, $U=(1 / V) \int_{V} u_{z} d v$, we define the dimensionless variables $u_{z}^{\prime}=u_{z} / U, z^{\prime}=z / L$, and $r^{\prime}=r / b$ and write Eq. (1) in the dimensionless form

$$
-\epsilon \Delta_{\theta}-f \mathrm{Ca}+\mathrm{Bo}=0 .
$$

In Eq. (4) we have used Eq. (3) and we have defined the dimensionless numbers

$$
\begin{aligned}
& \mathrm{Bo}=\frac{\rho g b^{2}}{\gamma(d-1)}, \quad \mathrm{Ca}=\frac{\mu U}{\gamma}, \\
& \epsilon=\frac{b}{L}, \quad \Delta_{\theta}=\cos \theta_{R}-\cos \theta_{A},
\end{aligned}
$$

and the shape function

$$
f\left(\theta_{A}, \theta_{R}, \mathrm{Ca}, \mathrm{Bo}, \epsilon\right)=\left.\int_{z_{A}^{\prime}}^{z_{R}^{\prime}} \frac{\partial u_{z}^{\prime}}{\partial r^{\prime}}\right|_{r^{\prime}=1} d \zeta>0,
$$

where $z_{A}^{\prime}$ and $z_{R}^{\prime}$ are the dimensionless positions of the advancing and the receding contact lines, respectively. Note that Eqs. (4)-(6) are valid for two-dimensional (2D) and 


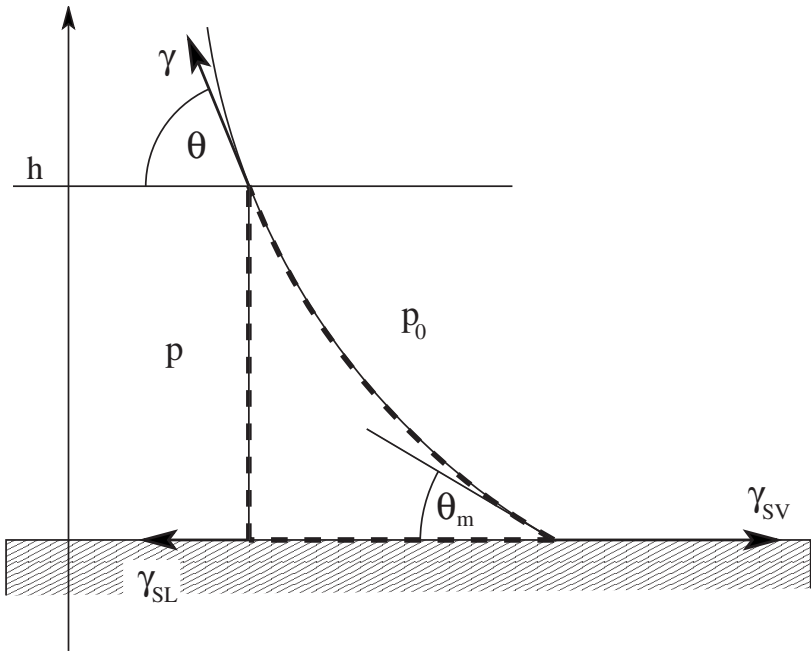

(a)

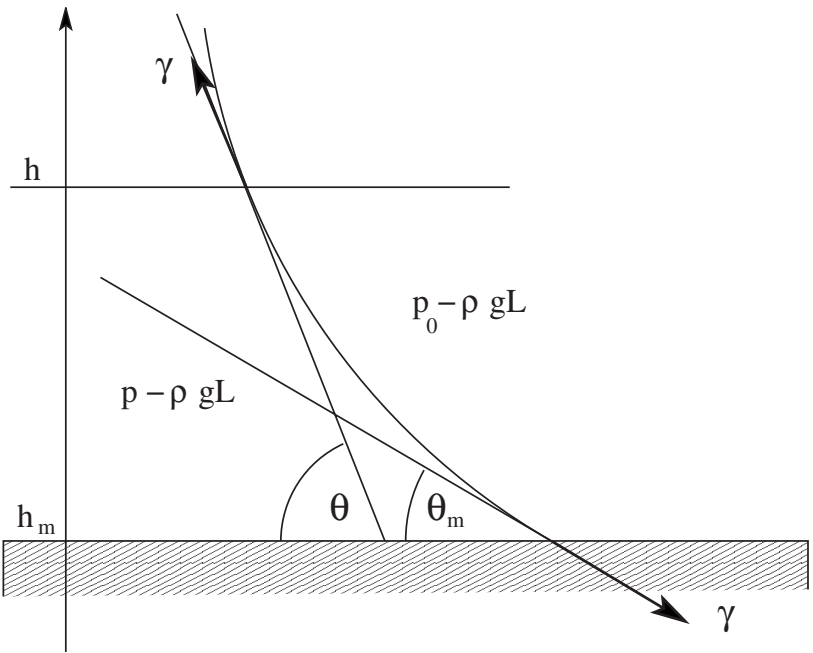

(b)

FIG. 2. (a) Surface tension and pressures acting on a wedge of height $h$; the resultant of these forces is balanced by the friction exerted on the wedge by the solid wall. (b) Representation of the microscopic contact angle, $\theta_{m}$, defined at the a distance $h_{m}$ from the wall at which the hydrodynamic description fails, and the macroscopic contact angle defined at a distance $h>h_{m}$.

three-dimensional problems with $d=\{1,2\}$ referring to the space dimensionality.

The first term in Eq. (4) represents microscopic hysteresis; the second term is the dimensionless viscous drag; and the third term the dimensionless body force. The shape function, $f$, expresses the effect of the velocity distribution on the tangential stress at the wall, Eq. (6). Since meniscus deformation modifies the velocity field, $f$ is a function of $\mathrm{Ca}$, Bo, and $\epsilon$, and the relationship between dimensionless velocity, $\mathrm{Ca}$, and the dimensionless weight, $\mathrm{Bo} / \epsilon$, is in general nonlinear. On the contrary, the shape of the menisci does not affect the capillary drag, which depends on the microscopic contact angle hysteresis only, Eq. (3). Equation (4) is exact (within the limits of validity of the hydrodynamic theory) and demonstrates that there is no capillary drag of hydrodynamic origin (i.e., due to hydrodynamic deformation of the interface) because only the viscous term depends on the slug velocity. If the there is no hysteresis of the microscopic contact angle $\left(\Delta_{\theta}=0\right)$, the additional drag with respect to Poiseuille flow is due to viscous forces only, Eq. (6).

\section{SHAPE FUNCTION AND VELOCITY FIELD}

In this section we restrict the analysis to the case in which $\mathrm{Bo}, \mathrm{Ca}$, and $\epsilon$ are small and we estimate the behavior of the shape function at leading order. Note that small Bo does not imply that gravity is negligible: the dimensionless weight is $\mathrm{Bo} / \epsilon$, and $\epsilon$ is also small.

\section{A. Interface shape near the contact line}

The shape of the interface near the contact line is described by the classical Voinov-Cox model. ${ }^{11,12}$ Here, we apply Voinov's analysis ${ }^{11}$ to the excess pressure over hydrostatic, i.e., $p-\rho g L$, rather than to the pressure itself and the uniform excess pressure in the gas becomes $p_{0}-\rho g L$ (Fig. 2). This is appropriate as long as the weight of the wedge itself is negligible (i.e., as long as $\operatorname{Bo}\left(h_{w}^{2} / b^{2}\right) \ll 1$ where $h_{w}$ is the height of the wedge). Relating the pressure at the free surface in a wedgelike geometry ${ }^{17}$ to the curvature of the free surface one obtains ${ }^{11,12}$

$$
\psi(\theta)-\psi\left(\theta_{m}\right):=\int_{0}^{\theta} \frac{d \phi}{c(\phi)}-\int_{0}^{\theta_{m}} \frac{d \phi}{c(\phi)}= \pm \mathrm{Ca} \ln \left(h / h_{m}\right),
$$

where $\theta=\theta(h)$, which describes the interface shape, is the macroscopic apparent contact angle measured at distance $h$ from the solid wall [Fig. 2(b)]; $\theta_{m}$ is the microscopic contact angle defined at a distance from the solid, $h_{m}$, comparable with the molecular size below which the continuum (hydrodynamic) description does not apply; plus and minus on the right hand side apply to the advancing, $\theta_{m}=\theta_{A}$, and receding, $\theta_{m}=\theta_{R}$, angles, respectively. If gas viscosity is negligible, we have

$$
c(\theta)=\frac{2 \sin \theta}{\theta-\sin \theta \cos \theta} \approx \frac{3}{\theta^{2}},
$$

where the approximation introduces an error smaller than $1 \%$ if $\theta<3 \pi / 4,{ }^{11}$ and the meniscus shape in the vicinity of the contact line is defined by

$$
\theta(h)=\left[\theta_{m}^{3} \pm 9 \mathrm{Ca} \ln \left(h / h_{m}\right)\right]^{1 / 3} .
$$

Equation (9) implies that there is a maximum velocity allowed for the receding contact angle, $\mathrm{Ca}_{\mathrm{cr}}$, which must remain positive or equal to zero. ${ }^{3,11}$ If the capillary number is increased further, no solution for a receding contact angle exists and a wetting film is left behind the slug (LandauLevich film ${ }^{3,11,18}$ ). 


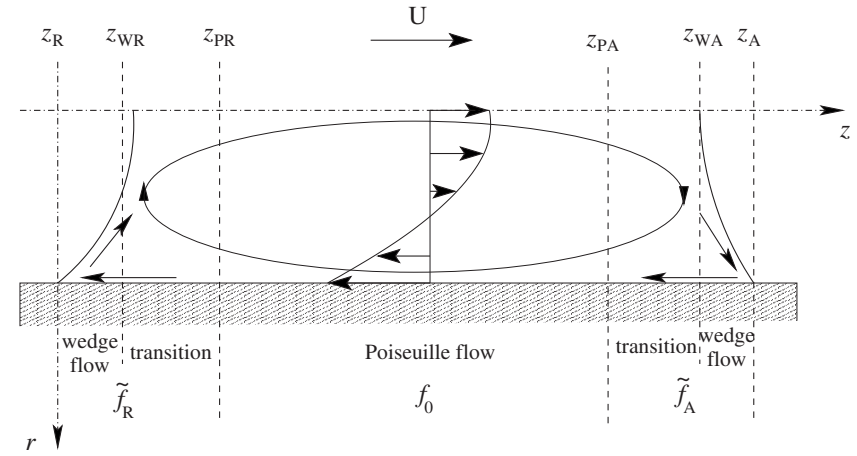

FIG. 3. Schematic representation of the velocity field in the half slug. The arrows indicate the velocity with respect to the center of mass of the slug, which translates with velocity $U>0$. The central region is characterized by a parabolic velocity profile (Poiseuille flow, $f_{P}$ ); the advancing and receding regions $\left(\tilde{f}_{A}\right.$ and $\tilde{f}_{R}$, respectively) are characterized by proper wedge-flow regions and regions of transition from Poiseuille to wedge flow.

\section{B. Viscous friction in the non-Poiseuille regions}

In the case of Poiseuille flow (infinite slug), the velocity profile is parabolic, $u_{z}^{\prime}=-d+1 / 2\left(r^{\prime 2}-1\right)$, and the shape function is $f=f_{0}=d+1$, where $d$ is the problem dimension. In a finite liquid slug, the parabolic velocity profile is perturbed in the vicinity of the menisci where capillary forces resist deformation of the interface. We define three distinct regimes (Fig. 3): a Poiseuille flow regime in the internal region, $z^{\prime} \in\left(z_{P R}^{\prime}, z_{P A}^{\prime}\right)$; a wedge-flow regime in the regions adjacent to the contact lines, $z^{\prime} \in\left(z_{R}^{\prime}, z_{W R}^{\prime}\right)$ and $z^{\prime} \in\left(z_{W A}^{\prime}, z_{A}^{\prime}\right)$, where the interface is described by the Voinov-Cox model (Sec. III A); and a transition regime, $z^{\prime} \in\left(z_{W R}^{\prime}, z_{P R}^{\prime}\right)$ and $z^{\prime}$ $\in\left(z_{P A}^{\prime}, z_{W A}^{\prime}\right)$. In the non-Poiseuille regions, streamlines are bent by capillary force and the resulting flow displays a typical rolling motion. ${ }^{19}$ Estimating the dimensionless length of those regions by $\epsilon$, we write

$$
f=f_{0}+\epsilon \tilde{f}=f_{0}+\epsilon\left(\tilde{f}_{w}+\tilde{f}_{t}\right)
$$

where $\tilde{f}=\tilde{f}_{w}+\tilde{f}_{t}$ accounts for the additional viscous friction due to streamline bending in the wedges, $\tilde{f}_{w}$, and in the transition regions, $\tilde{f}_{t}$.

If $\mathrm{Ca} \ll 1$ the meniscus shape near the capillary axis is a spherical cap independent (at leading order) of velocity, i.e., $\tilde{f}_{t}=O\left(\mathrm{Ca}^{0}\right)$. As we approach the wall, however, viscous effects become important due to the very large friction near the contact line, and the interface is deformed. If the free surface, $\theta(h)$, changes weakly with the distance from the solid surface, i.e., $(h / \theta)|(d \theta / d h)| \ll 1$, the classic solution of Huh and Scriven ${ }^{17}$ can be employed to compute the dissipation in the wedge region. ${ }^{11}$ This yields

$$
\tilde{f}_{w}=\int_{h_{m}^{\prime}}^{h_{w}^{\prime}} c(\theta) \sin (\theta) \frac{1}{\eta} d \eta,
$$

where $\theta$ depends on the distance from the wall through Eq. (9) and $h_{w}^{\prime}$ is the dimensionless wedge height. For finite contact angles, the leading order of $\tilde{f}_{w}$ can be determined by expanding $\theta$, Eq. (9), in powers of $\mathrm{Ca}$, i.e.,

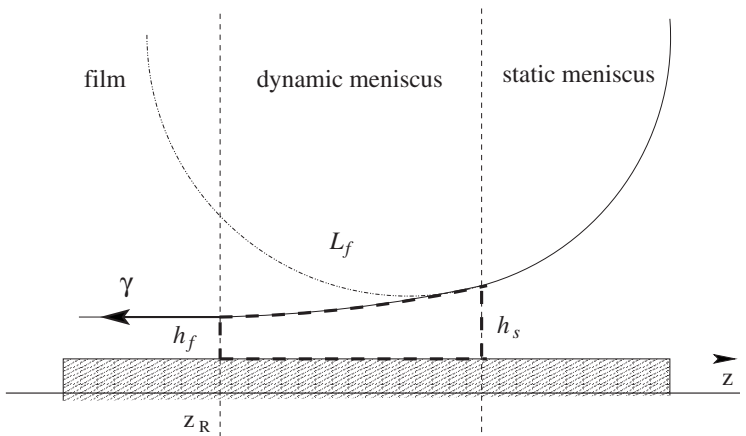

FIG. 4. The Landau-Levich flow in the receding meniscus: static film; dynamic meniscus (wedge flow); and static meniscus (transition region). The presence of the film induces a zeroth-order capillary drag, $\Delta_{\theta}=1-\cos \theta_{A}$, and remove the contact line singularity. The dissipation in the dynamic meniscus can be computed replacing $h_{m}$ by $h_{f}$ in Eq. (13) and recalling that the film height at the beginning of the static meniscus is $h_{w}=2.78 h_{f}$ (Ref. 20), which yields $\tilde{f}_{w, R}=2.20$.

$$
\tilde{f}_{w}=c\left(\theta_{m}\right) \sin \left(\theta_{m}\right) \ln \left(h_{w} / h_{m}\right)+O(\mathrm{Ca}) .
$$

If the contact angle is infinitesimal, $\theta_{m}=o(\mathrm{Ca})$, we first expand $\theta$ in power $\theta_{m}$, then in power $\mathrm{Ca}$, which leads to

$$
\tilde{f}_{w}=\frac{3}{2} \frac{3^{1 / 3}}{\mathrm{Ca}^{1 / 3}}\left[\ln \left(h_{w} / h_{m}\right)\right]^{2 / 3}+O\left(\mathrm{Ca}^{1 / 3}\right) .
$$

\section{Receding meniscus in presence of a trailing film}

When $\mathrm{Ca}>\mathrm{Ca}_{\mathrm{cr}}$, the film left behind the slug removes the singularity at the contact line and modifies the pressureforce resultant (Fig. 4). If the mass loss is negligible, the slug velocity can be considered quasisteady and the force balance expressed in Eq. (4) can be used. However, since the film is considered at rest, the position of the receding contact line,

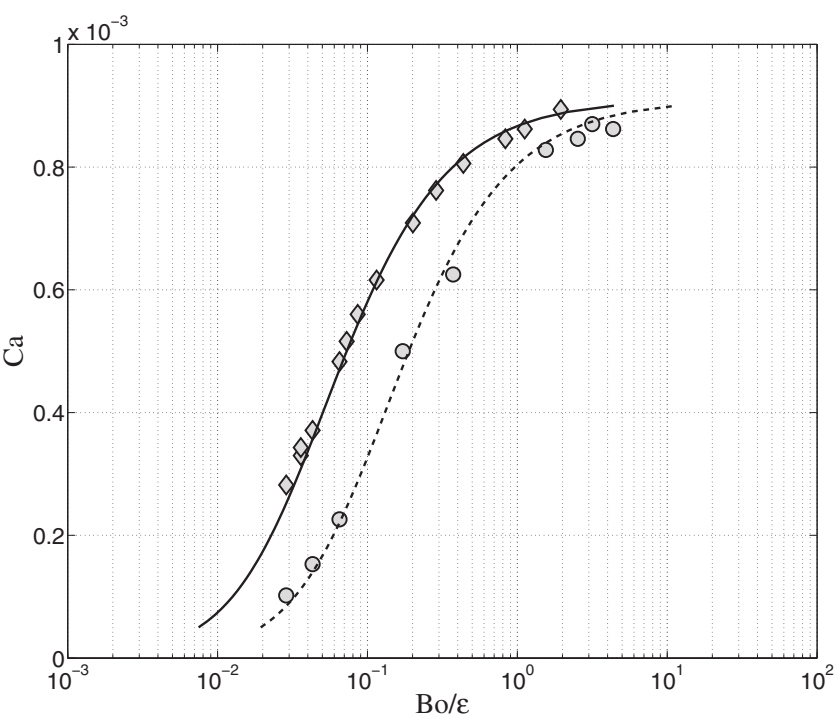

FIG. 5. The dimensionless velocity, $\mathrm{Ca}$, as a function of the dimensionless weight, Bo/ $\epsilon$, for the experiments of Bico and Quéré (Ref. 13): dry tube (circles) and prewetted tube (diamonds). The dry-tube data are fitted by Eq. (17) with $\alpha=13$ (dashed line); The prewetted-tube data are fitted by Eq. (17) with $\alpha=5.2$ (solid line). 


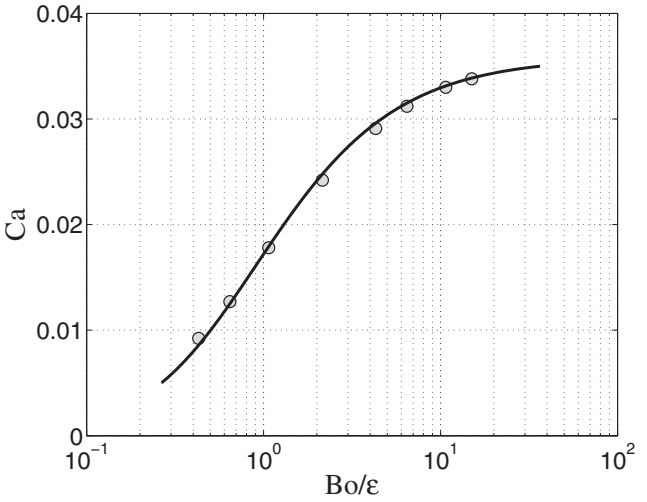

(a)

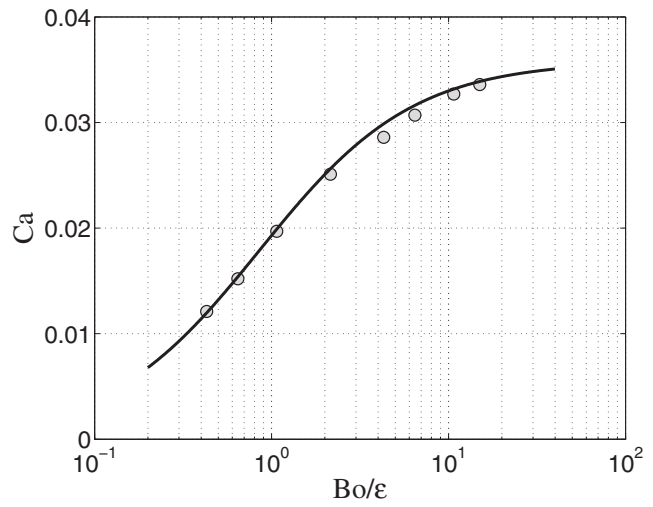

(c)

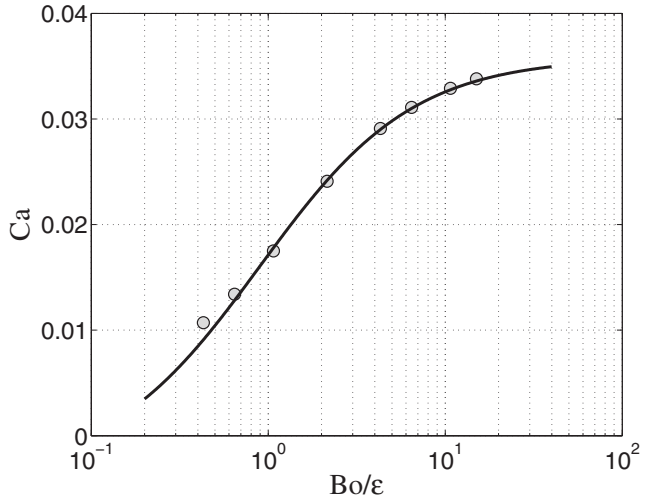

(b)

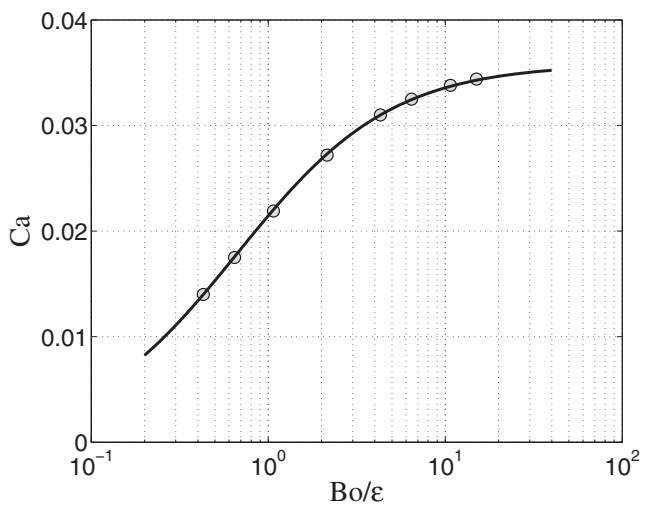

(d)

FIG. 6. Dimensionless velocity, Ca, as a function of the dimensionless weight, $\mathrm{Bo} / \epsilon$, for different static contact angles: (a) $\theta_{m}=0^{\circ}$, numerical simulations (circles) and the fit obtained with Eq. (17) and $\alpha=7.8$ (solid curve); (b) $25^{\circ}$, data are fitted by Eq. (15) with $\beta=25$ and $\Delta_{\theta}=1-\cos 25^{\circ}$; (c) $40^{\circ}$, data are fitted by Eq. (15) with $\beta=23.9$ and $\Delta_{\theta}=0$; and (d) $90^{\circ}$, data are fitted by Eq. (15) with $\beta=18.7$ and $\Delta_{\theta}=0$.

$z_{R}$, is defined as the point at which the static film begins (Fig. 4). The presence of the film causes the apparent contact angle at $z_{R}$ to drop to zero (Fig. 4). As shown in Appendix, the leading order effect of this transition is the appearance of a zeroth-order capillary drag, $\Delta_{\theta}=1-\cos \theta_{A}$, if the advancing contact angle is finite; whereas for infinitesimal advancing contact angles $\Delta_{\theta}=0$ and the leading order term is due to the dissipation in the dynamic meniscus. The latter is estimated replacing $h_{m}$ by the film thickness $h_{f}$ in Eq. (13) and recalling that the film height at the beginning of the static meniscus is $h_{w}=2.78 h_{f} .{ }^{20}$ This yields the shape function $\tilde{f}_{w, R}=2.20$ for the dynamic receding meniscus.

\section{SCALING LAWS FOR A SLUG FALLING AT CONSTANT VELOCITY}

Substituting Eq. (10) into Eq. (4), we obtain

$$
\left(\tilde{f} \mathrm{Ca}+\Delta_{\theta}\right) \epsilon=\mathrm{Bo}-f_{0} \mathrm{Ca} .
$$

For very long slugs, $\epsilon \ll 1$, the effect of the menisci (left hand side) is negligible and the dissipation approaches Poiseuille dissipation $\left(\mathrm{Ca}=\left(\mathrm{Bo} / f_{0}\right)\right)$. For finite slugs, however, the effects of the menisci must be taken into account. Considering the increase in viscous friction, $\widetilde{f}$, at the leading order in $\mathrm{Ca}$ for a finite microscopic contact angle, i.e., $\tilde{f}=\beta+O(\mathrm{Ca})$, and rearranging Eq. (14) we obtain

$$
\mathrm{Ca}=\frac{\mathrm{Bo} / \epsilon-\Delta_{\theta}}{f_{0} / \epsilon+\beta} .
$$

The parameter $\beta$ depends on the streamline geometry in the non-Poiseuille regions and includes the contribution of both wedge-flow and transition regions; for practical purposes $\beta$ may be regarded as a fitting parameter. As previously observed, the capillary drag, $\Delta_{\theta}$, is nonzero only in presence of microscopic contact angle hysteresis, or if transition to Landau-Levich flow occurs. Equation (15) predicts a different behavior of the velocity if the weight of the slug, $\mathrm{Bo} / \epsilon$, is increased by increasing the slug length or by increasing the liquid density or the acceleration: the relationship between velocity and weight is linear if Bo is varied while keeping $\epsilon$ constant, but it is nonlinear if Bo is kept constant while $\epsilon$ is varied. The latter case is qualitatively similar to the problem of a partially wetting drop sliding down a tilted surface, where acceleration is controlled by varying the inclination angle. For this problem, Podgorski et al. ${ }^{21}$ postulated a linear relationship between velocity and weight. Our Eq. (4) has two additional parameters, $\epsilon$ and $f$, that account for liquidshape effects and allow describing a nonlinear relationship.

For infinitesimal contact angles the contribution of the non-Poiseuille regions to the shape function is dominated by the flow in the wedges, Eq. (13), which yields $\tilde{f}=\alpha \mathrm{Ca}^{-1 / 3}$ $+\beta+O\left(\mathrm{Ca}^{1 / 3}\right)$. The coefficient $\alpha$ represents the contributions 

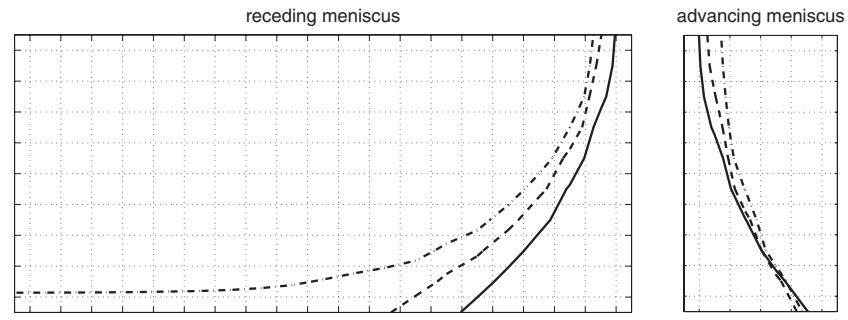

FIG. 7. The shape of the advancing (right) and receding (left) menisci obtained with the VOF method for a partially wetting liquid $\left(\theta_{m}=40^{\circ}\right)$. Shown are the half-meniscus shapes of three slugs of dimensionless size $1 / \epsilon=4$ (solid line), 20 (dashed line), and 40 (dashed-dotted line).

of both the wedgelike region near the advancing contact line and the dynamic receding meniscus, whereas $\beta$ describes the increase in viscous dissipation due to streamline bending in the transition regions (static menisci). Substituting $\widetilde{f}$ into Eq. (14) we obtain

$$
\left(\alpha \mathrm{Ca}^{2 / 3}+\beta \mathrm{Ca}\right) \epsilon=\mathrm{Bo}-f_{0} \mathrm{Ca},
$$

where we have set $\Delta_{\theta}=0$, being $\theta_{m} \ll 1$. Solving for $\epsilon$ we can write

$$
\epsilon=\frac{\mathrm{Bo}-f_{0} \mathrm{Ca}}{\alpha \mathrm{Ca}^{2 / 3}+\beta \mathrm{Ca}} \approx \frac{\mathrm{Bo}-f_{0} \mathrm{Ca}}{\alpha \mathrm{Ca}^{2 / 3}} .
$$

The approximation in Eq. (17) has the same velocity dependency as the equation used by Bico and Quéré ${ }^{13}$ to fit several experimental measurements of steady velocity of a silicone-oil (Rhodorsil 47v20) slug falling down a glass tube of radius $b=127 \mu \mathrm{m}$ under complete-wetting conditions. The experimental data of Ref. 13 are reproduced in dimensionless form in Fig. 5. The dry-tube data are fitted by the theoretical curve in Eq. (17) with $\alpha=13$ (Fig. 5). The fitting parameter is expected to be of order $\alpha \mathrm{Ca}^{2 / 3}=\left(\tilde{f}_{w, A}+\tilde{f}_{w, R}\right) \mathrm{Ca}=13.1 \mathrm{Ca}^{2 / 3}$, where the wedge shape function, Eq. (13), has been estimated by assuming $h_{w}=b$ and $h_{m}=1.5 \mathrm{~nm}$ (the typical molecular size obtained of the silicone oil employed, which has molar mass $1500 \mathrm{~g} / \mathrm{mol}$ and density $950 \mathrm{~kg} / \mathrm{m}^{3}$ ). Although we did not address the prewetted-tube case-throughly investigated by Jensen $^{22}$-we observe that the theoretical curve derived for small contact angles also fits these data with $\alpha=5.2$.

\section{NUMERICAL EVALUATION OF THE SCALING LAWS}

In this section, we evaluate the accuracy of the scaling laws in case of partial wetting and in presence of contact angle hysteresis by means of numerical simulations. We also verify the prediction of a linear relationship between velocity and weight when Bo is varied at constant $\epsilon$.

\section{A. The Volume of Fluid (VOF) method}

The numerical simulations are performed with the RIPPLE code, ${ }^{23}$ which has been modified to account for contact angle hysteresis. ${ }^{24,25}$ RIPPLE is a $2 \mathrm{D}$ code for modeling incompressible fluid flow with surface tension. The algo- rithm consists of two main steps: first the incompressible Navier-Stokes equations governing liquid flow are solved on a Cartesian grid by a two-step projection method (see, e.g., Ref. 26) and a conservative velocity field is obtained; then the velocity field is used to advect the free surface by a VOF method. ${ }^{27,28,23}$ In the VOF method the volume fraction of the liquid phase in each cell (color function) is tracked rather than the interface itself. Once the color function has been advected, the free surface is reconstructed by the algorithm of Youngs. ${ }^{29}$ Surface tension is implemented through the Continuum Surface Force (CSF) model, ${ }^{28}$ which transforms the surface force into a body force acting on the cells in which the color function takes a value between zero and one. Interactions with solid walls are modeled by prescribing the contact angle as boundary condition for the free interface orientation at the solid walls. A simple hysteresis model has been implemented, ${ }^{24,25}$ which allows prescribing different advancing, receding and equilibrium contact angles. We refer to Refs. 23 and 24 for further implementation details.

\section{B. Numerical results}

First, we consider slugs of increasing lengths, $1 / \epsilon$, falling down a capillary tube under different wetting conditions and neglect hysteresis $\left(\theta_{A}=\theta_{R}=\theta_{m}\right)$. The computational domain is discretized in a regular Cartesian grid with cell size $\delta=b / 10$. The dimensionless velocity, $\mathrm{Ca}$, is plotted, in Fig. 6 , as a function of dimensionless weight, $\mathrm{Bo} / \epsilon$, for $\theta_{m}=0^{\circ}, 25^{\circ}$, $40^{\circ}$, and $90^{\circ}$. The complete-wetting data are well fitted by Eq. (17) with $\alpha=7.8$, which is in agreement with the expected value, i.e., $\tilde{f}_{w} \approx 7$, where $h_{w} / h_{m}=b /(\delta / 2)$ has been used to estimate $\tilde{f}_{w}$, Eq. (12). (Note that the grid size is used to estimate wedge dissipation: the cutoff in Eq. (7) is determined by the numerical resolution rather than by the molecular scale, which is not explicitly resolved by the simulations.)

The partial-wetting data are fitted with Eq. (15). For $\theta_{m}=25^{\circ}$ we set $\Delta_{\theta}=1-\cos \theta_{m}$ since a film is left behind the slug, whereas for the other two series of data $\left(\theta_{m}=40^{\circ}\right.$ and $90^{\circ}$ ) we set $\Delta_{\theta}=0$. The theoretical curves accurately describe the numerical results. Deviations are observed only for $\theta_{m}=40^{\circ}$ when $\mathrm{Bo} / \epsilon>4$. This is expected because for slugs of this size, $1 / \epsilon>20$, transition to Landau-Levich flow occurs, as demonstrated by Fig. 7, which shows the shape of advancing and receding menisci for slugs of different lengths $1 / \epsilon=4,20$, and 40 . The behavior agrees with the experimental observations of contact line dynamics and demonstrates that the numerical code is capable of simulating, at least qualitatively, the interactions among capillary, gravity, and viscous forces in the vicinity of the menisci.

The fitting parameters obtained by linear regression of the numerical data are reported in Table I together with the contribution of the shape functions, $2 f_{w}$, which are estimated using the tube radius, $b$, as macroscopic length and the half grid size, $\delta / 2$, as cutoff length in Eq. (13). (Only data for partially wetting slugs and velocity below the critical value for transition to Landau-Levich flow, i.e., $\mathrm{Ca}<\mathrm{Ca}_{\mathrm{cr}}$, have been considered). The ratio of the expected wedge contribution to total non-Poiseuille dissipation, $2 f_{w} / \beta$, is plotted in Fig. 8 as a function of the contact angle. The numerical 
TABLE I. The fitting parameter, $\beta$, for different contact angles, $\theta_{m}$; also shown is an estimate of the shape function associated with dissipation in the advancing and receding wedges, $2 f_{w}$, which is calculated using the tube radius, $b$, as macroscopic length and the half grid size, $\delta / 2$, as cutoff length.

\begin{tabular}{lrrrrrr}
\hline \hline & \multicolumn{7}{c}{$\theta_{m}$} \\
\cline { 2 - 7 } & \multicolumn{1}{c}{90} & \multicolumn{1}{c}{ (50 } & 60 & 45 & 40 & 30 \\
\hline$\beta$ & 18.7 & 24.0 & 23.5 & 23.4 & 23.9 & 26.4 \\
$2 f_{w}$ & 5.9 & 8.1 & 11.3 & 16.1 & 18.5 & 25.4 \\
\hline \hline
\end{tabular}

simulations indicate that the fraction of dissipation due to wedge-flow sensibly decreases for large contact angles, and suggest that bending of streamlines in the region of transition from wedge to Poiseuille flow sensitively contributes to viscous friction for large wetting angles. A numerical test with static contact-angle hysteresis $\left(\theta_{A}=65^{\circ}\right.$ and $\left.\theta_{R}=35^{\circ}\right)$ is shown in Fig. 9. The computational domain was discretized into a regular Cartesian grid with cell size $\delta=b / 10$. Also in this case an excellent agreement between numerical results and Eq. (15) is obtained.

Finally, in order to demonstrate the predictive capability of the derived scaling law, Eq. (15), we consider two scenarios: in the first, Bo is fixed and the slug length, $1 / \epsilon$, is varied; in the second, the slug length is constant, $1 / \epsilon=4$, and Bo is varied (e.g., by varying liquid density or acceleration). The computational domain is discretized in a regular Cartesian grid with cell size $\delta=b / 20$. The numerical data points obtained for $\theta_{m}=90^{\circ}$ and $\theta_{m}=45^{\circ}$ are plotted in Fig. 10 . The results obtained by varying the Bond number at fixed slug volume clearly show a linear relationship between $\mathrm{Ca}$ and Bo. By fitting these results with Eq. (15), we obtain the parameter $\beta$ which is then used to predict velocity changes when the slug length was increased at constant Bo. As seen in Fig. 10, the same value of $\beta$ fits both numerical data sets. This numerically proves the validity of Eq. (15) and demonstrates that $\tilde{f}$ is of order $O\left(\mathrm{Ca}^{0}\right)$, hence independent of the velocity at leading order. [Note that for $\theta_{m}=45^{\circ}$ the capillary

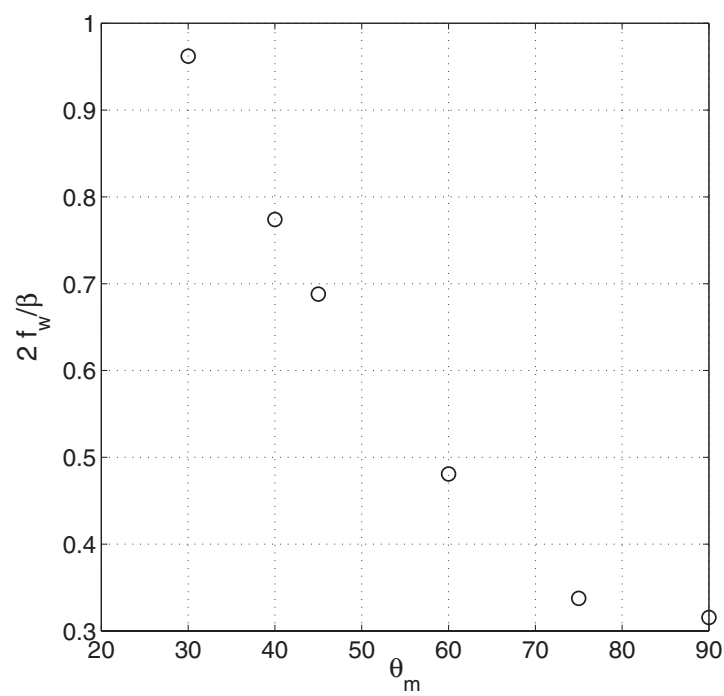

FIG. 8. The ratio of the expected wedge contribution to total non-Poiseuille dissipation, $2 f_{w} / \beta$, as a function of the contact angle, $\theta_{m}$. (Data from Table I.) number obtained for Bo/ $\epsilon \sim 2.1$ lies below the theoretical curve because transition to Landau-Levich flow has occurred, Fig. 10(b).] The different relationships between velocity and weight observed in the two scenarios are due to the different impact of bulk dissipation. If the length is varied, bulk dissipation increases with slug weight, which results in a nonlinear relationships converging to Poiseuille velocity for infinite slugs. If the length is kept constant, the ratio between bulk dissipation and non-Poiseuille dissipation does not change (the shape function is constant) and the relationship is linear.

\section{DISCUSSION AND CONCLUSIONS}

The steady velocity of liquid slugs falling down a capillary tube is lower than predicted by Poiseuille's law due to the presence of menisci that generate an additional drag force. Previous studies addressing similar problems have attributed the origins of this force to modification of the capillary pressure acting on the menisci, whereas the main source of friction remains the Poiseuille-like viscous dissipation in the bulk liquid. ${ }^{13-15,24}$ We argue, however, that the presence of menisci bends streamlines in a region close to the interface, thereby affecting the form of viscous dissipation, which will differ from Poiseuille's.

To identify the physical origin of the additional drag, we calculate the capillary-force resultant acting on an interface

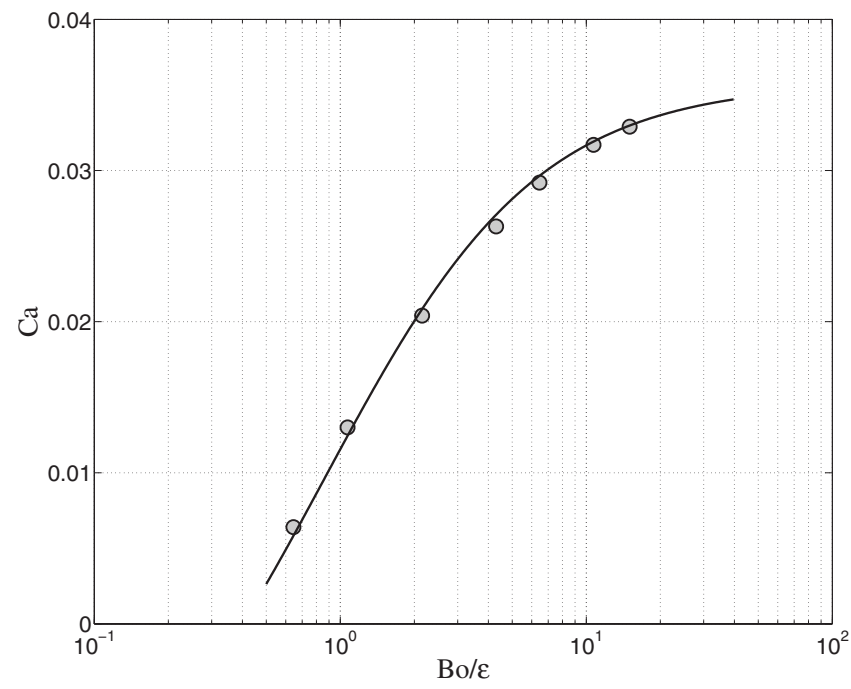

FIG. 9. Dimensionless velocity, $\mathrm{Ca}$, as a function of the dimensionless weight, $\mathrm{Bo} / \epsilon$, for a liquid displaying static contact angle hysteresis, i.e., $\theta_{A}=65^{\circ}$ and $\theta_{R}=35^{\circ}$. The data are fitted by Eq. (15) with $\beta=24$ and $\Delta_{\theta}=\cos 35^{\circ}-\cos 65^{\circ}=0.4$. 

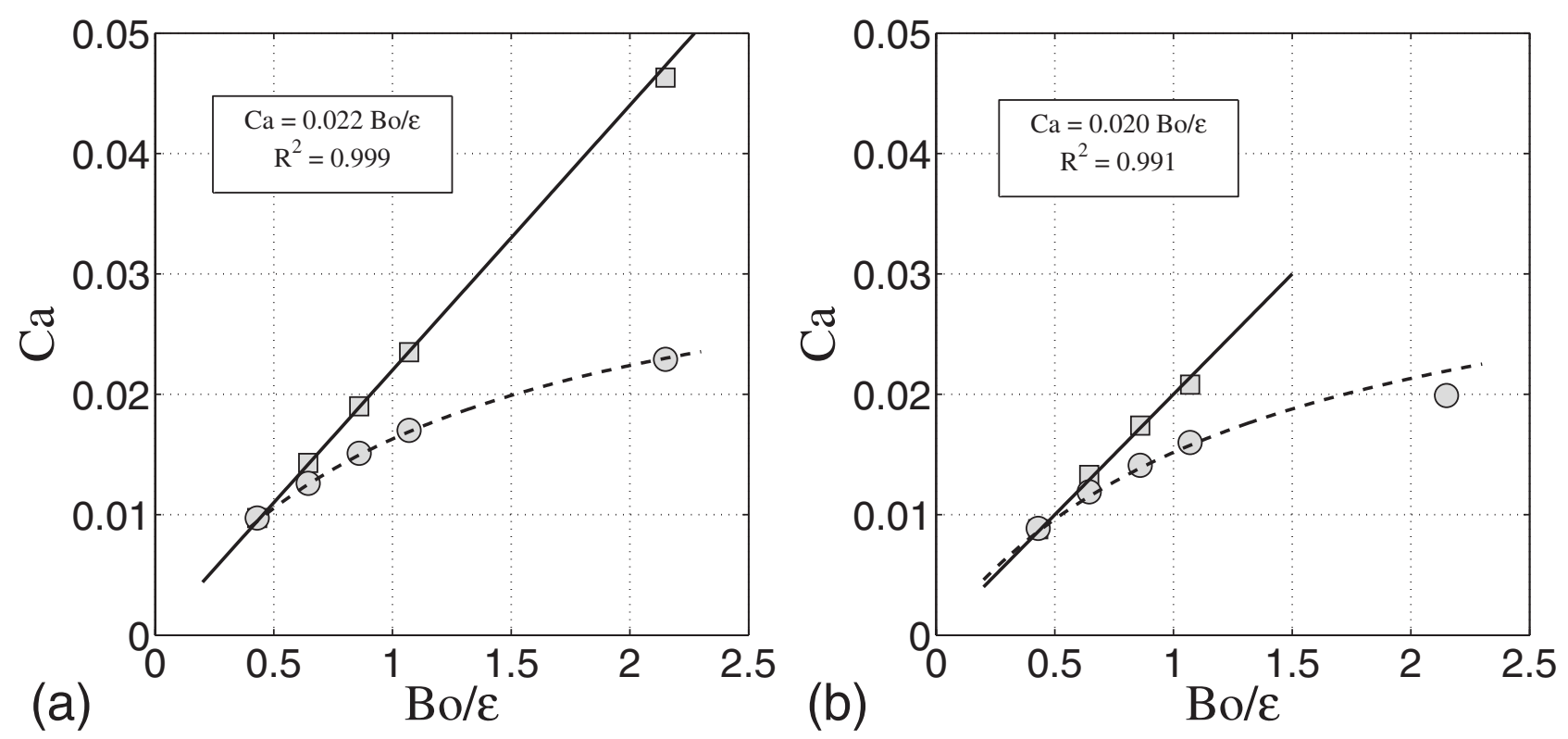

FIG. 10. Dimensionless velocity, Ca, as a function of the dimensionless weight, Bo/ $\epsilon$, for different static contact angles: (a) $\theta_{m}=90^{\circ}$; (b) $\theta_{m}=45^{\circ}$. Both the values for increasing Bo at constant $1 / \epsilon$ (squares) and for increasing 1/ $\epsilon$ at constant Bo (circles) are plotted. Also shown is the linear formulas that fit the data obtained varying Bo, and the corresponding linear-correlation coefficient, $R^{2}$.

of arbitrary shape using Frenet's formulas. This leads to Eq. (3), whose interpretation is straightforward: the resultant of surface tension acting on the interface can be calculated as the integral over the interface boundary, the contribution of the internal points vanishing in virtue of the action-reaction principle. ${ }^{30}$ Since this procedure only relies on the hypothesis that capillary pressure is proportional to the interface curvature, the interface boundary can be located at a distance from the wall at which the hydrodynamic description fails and the capillary drag is defined in terms of the microscopic contact angles, defined at a distance from the wall comparable to the molecular scale. These arguments demonstrate that the additional drag force is exclusively of viscous origin, while the resultant of the capillary forces is unaffected by hydrodynamic deformations of the menisci.

To represent the effects of meniscus deformation on viscous friction we have introduced a shape function which accounts for the effects of the velocity field in the slug, Eq. (6), and can describe a nonlinear dependence of the viscous drag on the mean velocity (or the capillary number, $\mathrm{Ca}$ ). The latter is the case of complete wetting for which we have derived an explicit scaling law by estimating the shape function at leading order in $\mathrm{Ca}$, Eq. (17). This law agrees with the equation derived by Bico and Quéré ${ }^{13}$ to relate velocity and slug length, which has been validated experimentally. Bico and Quéré $^{13}$ derived their equation assuming that the viscous term obeys Poiseuille dissipation and that the additional drag is due to capillary pressure acting on spherical menisci whose curvature follows Hoffman-Tanner's and Bretherton's laws at the advancing and receding menisci, respectively. The apparent capillary drag introduced by Bico and Quéré ${ }^{13}$ arises from the fact that the nonspherical part of the interface is not explicitly modeled, but parametrized in the apparent contact angle. However, it is evident from Eqs. (3) and (4) that the physical mechanism ultimately responsible for the additional drag is viscous dissipation.

The case of partial wetting leads to a different scaling law, Eq. (15). If the slug length, $1 / \epsilon$, is kept constant and the weight is varied by modifying the liquid density or the gravity acceleration (hence, modifying Bo), the relationship between dimensionless velocity, $\mathrm{Ca}$, and dimensionless weight, $\mathrm{Bo} / \epsilon$, is linear. This is due to the fact that the shape of the slug, which determines the viscous drag, is primarily determined by the surface tension. When Bo is kept constant and the slug length is varied, Eq. (15) predicts a nonlinear relationship between velocity and weight: by increasing the slug length both weight and dissipation increase. These results are supported by numerical simulations, which also suggest that wedge-flow dissipation alone cannot account for the entire additional drag and that the dissipation in the transition region (static meniscus) cannot be neglected. Note that in this case, a description of the additional drag in terms of modified capillary pressure is not possible because wedge dissipation, affecting the apparent contact angle, contributes only to a portion of the non-Poiseuille dissipation (and this portion decreases when the contact angle increases). This confirms that the additional drag force is of viscous origin and shows that in case of partial wetting the dissipation cannot be reduced to Poiseuille's due to the streamlines bending at the slug extremities.

\section{ACKNOWLEDGMENTS}

We are grateful to Hai Huang for his introduction to RIPPLE and for sharing with us his modified version of the code. 


\section{APPENDIX: PRESSURE RESULTANT IN PRESENCE OF A TRAILING FILM}

In this Appendix we derive the leading order modification of the pressure forces acting on the slug when transition to Landau-Levich flow occurs. We define the position of the receding contact line, $z_{R}$, as the point at which the static film begins, which yields zero apparent contact angle at $z_{R}$ (Fig. 4). Invoking Eq. (2) we write

$$
\int_{S} p n_{j} d s=\gamma \Delta_{\theta} \oint_{r=b} d l+\gamma W,
$$

where $\Delta_{\theta}=1-\cos \theta_{A}$ and $W$ accounts for the presence of the static film. If $d=2$, then $W=0$; if $d=3$, we have

$$
W=\oint_{r=b-h_{f}} d l-\oint_{r=b} d l-\kappa_{f} \int_{S_{f}} d s=\pi \frac{h_{f}^{2}}{b-h_{f}} \sim b \mathrm{Ca}^{4 / 3},
$$

where $S_{f}$ is the interface with the static film, and Bretherton's ${ }_{\text {law }}{ }^{20}$ has been used for the ratio of the film thickness to the capillary radius, $h_{f} / b \ll 1$. For finite advancing contact angles, the leading order in $\mathrm{Ca}$ is the zeroth-order capillary drag, $\Delta_{\theta}=1-\cos \theta_{A}$; for infinitesimal advancing contact angles, $\Delta_{\theta}=0$, the leading order term is due to the dissipation in the dynamic meniscus, which is $O\left(\mathrm{Ca}^{2 / 3}\right)$.

${ }^{1}$ L. D. Landau and E. M. Lifshitz, Fluid Mechanics (Pergamon, Oxford, 1959).

${ }^{2}$ L. H. Tanner, "The spreading of silicone oil drops on horizontal surfaces," J. Phys. D 12, 1473 (1979).

${ }^{3}$ P.-G. de Gennes, "Wetting: Statistics and dynamics," Rev. Mod. Phys. 57, 827 (1985).

${ }^{4}$ F. Brochard-Wyart and P. G. de Gennes, "Dynamics of partial wetting," Adv. Colloid Interface Sci. 39, 1 (1992).

${ }^{5}$ T. D. Blake and K. J. Ruschak, "A maximum speed of wetting," Nature (London) 282, 489 (1979).

${ }^{6}$ P. G. de Gennes, "Deposition of Langmuir-Blodgett layers," Colloid Polym. Sci. 264, 463 (1986).

${ }^{7}$ X. Chen, E. Rame, and S. Garoff, "The effects of thin and ultrathin liquid films on dynamic wetting," Phys. Fluids 16, 287 (2004).

${ }^{8}$ P. G. de Gennes, F. Brochard-Wyart, and D. Quéré, Capillary and Wetting Phenomena. Drops, Bubbles, Pearls, Waves (Springer-Verlag, New York, 2004).
${ }^{9}$ R. L. Hoffman, "A study of the advancing interface. 1. Interface shape in liquid-gas systems,” J. Colloid Interface Sci. 50, 228 (1975).

${ }^{10}$ M. Fermigier and P. Jenffer, "An experimental investigation of the dynamic contact angle in liquid-liquid systems," J. Colloid Interface Sci. 146, 226 (1991).

${ }^{11}$ O. V. Voinov, "Hydrodynamics of wetting," Fluid Dyn. 11, 714 (1976).

${ }^{12}$ R. G. Cox, "The dynamics of spreading of liquids on a solid surface. Part 1. Viscous flow," J. Fluid Mech. 168, 169 (1986).

${ }^{13}$ J. Bico and D. Quéré, "Falling slugs," J. Colloid Interface Sci. 243, 262 (2001).

${ }^{14}$ G. W. Su, J. T. Geller, J. R. Hunt, and K. Pruess, "Small-scale features of gravity-driven flow in unsaturated fractures," Vadose Zone J. 3, 592 (2004).

${ }^{15}$ M. I. Dragila and N. Weisbrod, "Parameters affecting maximum fluid transport in large aperture fractures," Adv. Water Resour. 26, 1219 (2003).

${ }^{16}$ D. J. Struik, Lectures on Classical Differential Geometry (Dover, New York, 1988).

${ }^{17}$ C. Huh and L. E. Scriven, "Hydrodynamic model of steady movement of solid/liquid/fluid contact line,” J. Colloid Interface Sci. 35, 85 (1971).

${ }^{18}$ L. Landau and B. Levich, "Dragging of a liquid by a moving plane," Acta Physicochimica URSS 17, 42 (1942).

${ }^{19}$ E. B. Dussan V and S. H. Davis, "On the motion of a fluid-fluid interface along a solid surface," J. Fluid Mech. 65, 71 (1974).

${ }^{20}$ F. P. Bretherton, "The motion of long bubbles in tubes," J. Fluid Mech. 10, 166 (1961).

${ }^{21}$ T. Podgorski, J.-M. Flesselles, and L. Limat, "Corners, cusps, and pearls in running drops," Phys. Rev. Lett. 87, 036102 (2001).

${ }^{22} \mathrm{O}$. E. Jensen, "Draining collars and lenses in liquid-lined vertical tubes," J. Colloid Interface Sci. 221, 38 (2000).

${ }^{23}$ D. B. Kothe, R. C. Mjolsness, and M. D. Torrey, "RIPPLE: A computer program for incompressible flows with free surfaces," Technical Report No. LA-12007-MS, Los Alamos National Laboratory, Los Alamos, NM, 1991.

${ }^{24}$ H. Huang, P. Meakin, and M. Liu, "Computer simulation of two-phase immiscible fluid motion in unsaturated complex fractures using a volume of fluid method," Water Resour. Res. 41, W12413 (2005).

${ }^{25}$ H. Huang, P. Meakin, M. Liu, and G. E. McCreery, "Modeling of multiphase fluid motion in fracture intersections and fracture networks," Geophys. Res. Lett. 32, L19402, DOI: 10.1029/2005GL023899 (2005).

${ }^{26}$ R. Peyret and T. D. Taylor, Computational Methods for Fluid Flow (Springer-Verlag, New York, 1983).

${ }^{27}$ C. W. Hirt and B. D. Nichols, "Volume of fluid (VOF) method for the dynamics of free boundaries," J. Comput. Phys. 39, 201 (1981).

${ }^{28}$ J. U. Brackbill, D. B. Kothe, and C. Zemach, "A continuum method for modeling surface tension," J. Comput. Phys. 100, 335 (1992).

${ }^{29}$ D. L. Youngs, Numerical Methods for Fluid Dynamics (Academic, New York, 1982), pp. 273-285.

${ }^{30}$ I. Lunati, "Young's law and the effects of interfacial energy on pressure at the solid-fluid interface," Phys. Fluids 19, 118105 (2007). 